\title{
Mixture Rasch Model with Main and Interaction Effects of Covariates on Latent Class Membership
}

\author{
Tugba Karadavut (id 1,*, Allan S. Cohen (id 2, Seock-Ho Kim (id 2 \\ ${ }^{1}$ Recep Tayyip Erdogan University, Faculty of Education, Cayeli, Rize, Turkey \\ ${ }^{2}$ The University of Georgia, Department of Educational Psychology (Quantitative Methodology), Athens, GA, \\ USA
}

\section{ARTICLE HISTORY}

Received: 21 February 2019

Revised: 17 June 2019

Accepted: 02 July 2019

\section{KEYWORDS}

Covariates,

Item response theory,

Latent class membership,

Latent covariates,

Mixture Rasch model

\begin{abstract}
Covariates have been used in mixture IRT models to help explain why examinees are classed into different latent classes. Previous research has considered manifest variables as covariates in a mixture Rasch analysis for prediction of group membership. Latent covariates, however, are more likely to have higher correlations with the latent class variable. This study investigated effects of including latent variables as covariates in a mixture Rasch model, in presence of and in absence of interactions between the covariates. Results indicated the latent and manifest covariates influenced latent class membership but did not have much influence on class ability means or class proportions. The influence was relatively higher for latent covariates compared to manifest covariates. The effects of the covariates on class membership and on item parameters were class specific. Substantial effects of covariates on item parameters yielded smaller standard errors for item parameter estimates. A significant interaction term also had an effect on the coefficients for predicting and explaining latent class membership.
\end{abstract}

\section{INTRODUCTION}

A mixture Rasch model (MRM; Rost, 1990) assumes the examinee population is comprised of a finite number of discrete latent classes and a Rasch model with different item parameters possible within each class. The latent class portion of the model accounts for qualitative differences among examinees by detecting latent classes. The Rasch model part of the model accounts for quantitative differences among examinees both within and between latent classes. The MRM by itself detects the latent classes, but it does not explain why these classes form. This is necessary in order to understand why examinees are classified into different latent classes. Once latent classes are detected, therefore, a next step is to characterize each class to better understand the differences between classes. One method used for providing more information about these differences is addition of a covariate to the model in order to improve modeling of the association between the covariate and the latent class membership (Bilir, 2009; Cho, Cohen, \& Kim, 2013; Choi, Alexeev, \& Cohen, 2015; Dai, 2013; Smit, Kelderman, \& van der Flier, 1999). 
Different approaches can be adopted for inclusion of a covariate in a mixture model depending on the type (e.g., item specific covariates that refer to items) and level of the covariate (e.g., within level latent class covariates that are used to predict the latent class membership for a specific level in a multilevel model), or the parameter of interest to be predicted by the covariate (e.g., latent class membership, ability). The approach of extending mixture item response theory (IRT) models to include a multinomial logistic regression model with a covariate is adopted in this study to predict the latent class membership by using the covariate (Cho et al., 2013; Dai, 2013). The covariate in these models can be used as prior information (e.g., as an auxiliary variable) to predict the posterior probabilities of latent class membership.

Incorporation of a covariate in a mixture IRT model has been shown to be useful for detection of the latent classes and also for characterizing differences between the latent classes (e.g., Bilir, 2009; Choi et al., 2015; Dai, 2013; Smit et al., 1999). Previous research has included a single manifest categorical variable as a covariate in the model. Manifest covariates are not always sufficiently informative, however, they tend to be only moderately related to the variable causing the latent classes to form. In a differential item functioning (DIF) context, for example, manifest grouping variables were not very helpful for explaining causes of between group differences (Cohen \& Bolt, 2005). In this study, we compare the effects of manifest and latent covariates with and without interactions on latent class membership. As an exploratory investigation, we tried to accomplish the purpose by presenting an application to data from the Program for International Assessment (PISA; OECD, 2013) mathematics literacy test.

In this paper, a finite mixture multinomial logistic regression structure with covariates was incorporated into a MRM for this purpose (cf. Cho et al., 2013). Latent covariates were expected to yield higher relationships with the latent class variable, because they were both obtained from examinee response data, albeit not from the same measures. This was expected to enhance the impact of the covariates on detection and subsequent characterization of the latent classes.

\section{METHOD}

\subsection{Mixture Rasch Model (MRM) and Mixture Rasch Model with a Covariate (MRM- Cov)}

Rost (1990) defined the probability of a correct response to item $i$ by examinee $j$ given that the examinee belongs to latent class $g$ as:

$$
\mathrm{P}\left(\mathrm{X}_{\mathrm{ii}}=1 \mid \theta_{\mathrm{i}}, \mathrm{g}\right)=\left(\frac{\exp \left(\theta_{\mathrm{i} \xi}-\mathrm{b}_{\mathrm{i} \xi}\right)}{1+\exp \left(\theta_{\mathrm{j} \xi}-\mathrm{b}_{\mathrm{i} \varepsilon}\right)}\right),
$$

where $\theta_{j g}$ is the examinee's ability in class $g, b_{i g}$ is the class specific item difficulty parameter, and $X_{i j}$ is the observed response of examinee $j$ to an item $i$. For model identification, $\sum_{\mathrm{i}=1}^{!} b_{\mathrm{i} \varepsilon}=0$ holds within each class. Bolt, Cohen, and Wollack (2002) noted that this norming constraint also makes $\theta \mathrm{g}$ comparable across classes and that the differences between the $\theta_{g}$ distributions can quantitatively explain the differences between the latent classes.

The MRM with a covariate (MRM-Cov) can incorporate a multinomial logistic regression model for $\pi_{g}$ in Equation 1 as follows:

$$
\operatorname{logit}\left(\pi_{j_{\xi}}\right)=\beta_{0}+\beta_{1} y_{j}
$$

or similarly,

$$
\pi_{\mathrm{j}\}}=\frac{\exp \left(\beta_{0}+\beta_{1} \mathrm{y}_{\mathfrak{j}}\right)}{\sum_{\mathrm{g}=1}^{\mathrm{G}} \exp \left(\beta_{0}+\beta_{1} \mathrm{y}_{\mathrm{j}}\right)},
$$


with the covariate $y_{j}$ as the predictor, where $\beta_{0 g}$ is the intercept, and $\beta_{1 g}$ is the covariate effect in latent class $g$. The intercept and covariate effects in one of the latent classes were both fixed to zero for model identification (Cho et al., 2013).

\subsection{Selection of Covariates}

Smit et al. (1999) describe use of collateral information that has strong association with the latent class variable resulting in smaller standard errors on parameter estimates, when an equal or even fivefold smaller sample size was used in a MRM. Selection of a covariate that has strong association with the latent class membership, however, also requires theoretical as well as statistical justification. In most testing situations, manifest collateral information (e.g., demographic or contextual information) is available, since this type of information can easily be obtained through simple questionnaires or reference to institutional records.

Manifest variables, unfortunately, are not necessarily very useful predictors of latent class membership as the association between a manifest variable and the variable causing latent classes to form is typically modest at best (Cohen \& Bolt, 2005). Further, the proportions of variance explained by these manifest variables are usually small even though they might be significant. Cohen and Bolt (2005) noted that latent variables, on the other hand, often have stronger relationships with the latent classes, thus providing more useful information regarding formation of the latent classes. Latent variables, however, typically require more complex substantive theories or statistical models for detection. In this study, we discuss using manifest and latent variables as covariates in the MRM analysis of PISA (OECD, 2013) mathematics literacy data. The student questionnaires from PISA provided collateral variables that were assessed for selection of latent as well as manifest covariates.

Strength of association between the covariates and the latent classes can be defined using bivariate probabilities of classification (Smit et al., 1999), or by using exponents of the coefficients (Dai, 2013). Two steps were used in the present study to determine appropriate latent covariates for incorporating in Equation 3: (1) the covariate selection, and (2) the MRMCov analysis with the selected covariates. In the covariate selection step, correlation coefficients were examined between candidate covariates and the latent variable of interest in order to determine the strength of association. In the second step, the exponents of the coefficients were determined as a measure of the association between the covariates and the latent classes in a MRM-Cov model. An empirical example is provided to demonstrate the two-step procedure for fitting a MRM-Cov model.

\subsection{Empirical Example: Use of Latent Covariates to Predict Latent Class Membership in a Mixture Rasch Model}

We illustrate this two-step procedure for selection and inclusion of covariates for predicting latent class membership in a MRM with two examples. The two examples included two different MRM-Cov models each including different combinations of latent and manifest covariates. The model in Study A included two covariates which did not have a significant interaction. The model in Study B included two covariates which did have a significant interaction. The purpose in these two studies was to gain insight about the effects of including more than one covariate in a MRM-Cov model on class membership in the presence of and in the absence of an interaction between the covariates. In addition, each study included a manifest and a latent covariate in order to compare their influence on the latent class membership.

\subsubsection{Data}

Data for the studies were taken from the 2012 edition of PISA (OECD, 2014) that assessed mathematics literacy as the main domain. Data from six English speaking countries $(N=1,372)$ were used to mitigate differences due to translations (e.g., Bonnet, 2002): Australia $\left(n_{1}=312\right)$, 
Canada $\left(n_{2}=447\right)$, United Kingdom $\left(n_{3}=289\right)$, Ireland $\left(n_{4}=117\right)$, New Zealand $\left(n_{5}=88\right)$, and the United States $\left(n_{6}=119\right)$. PISA 2012 provided non-cognitive measures for students including manifest (e.g., demographic information, number of books at home) and latent variables (e.g., attitudes). Mathematics-related variables (e.g., attitudes towards math, beliefs about math) were considered for use as latent covariates. Booklet 5 was used for this example from the 13 booklets used for PISA 2012, because it included only mathematics items and most of its items required higher levels of cognitive process (e.g., employ, interpret) (OECD, 2014).

\subsubsection{Selection of Manifest Covariates}

A mathematics achievement score was calculated by summing the dichotomous item scores from the mathematics literacy test for each student. Among the manifest variables available with the PISA 2012 data, 31 were evaluated for possible use as covariates. These candidate variables were regressed on the raw mathematics achievement scores to find the most significant manifest variable that explained the largest proportion of variance. The purpose of this analysis was to find the manifest variable that was the best predictor of the mathematics achievement given the data. Regression analysis suggested that number of books at home was the best predictor of the mathematics achievement score $(\mathrm{R}$-square $=.124)$. Average hours a student spend each week on homework predicted the second highest proportion of variance explained $(\mathrm{R}$-square change $=.031)$.

\subsubsection{Selection of Latent Covariates}

PISA 2012 included four non-cognitive measures considered to be outcomes of mathematics education: (1) mathematics-related attitudes, beliefs and motivation; (2) general school-related attitudes and behaviors; (3) motivation to learn; and (4) educational expectations (OECD, 2013). Of these variables, those specifically dealing with mathematics-related attitudes, beliefs and motivation were considered as potential covariates. The mathematics-related attitudes included student interest in mathematics and student willingness to engage in mathematics. Student interest in mathematics included interest in mathematics at school, and intentions for further study in mathematics and in mathematics related careers. The willingness to be engaged was measured as "emotions of enjoyment, confidence and (lack of) mathematics anxiety, and the self-related beliefs of self-concept and self-efficacy" (OECD, 2013, p. 42). A mathematicsrelated attitude variable was considered as a potential covariate by combining the scales of the variables that comprised the mathematics-related attitudes. However, the scales of these variables were quite different for some of the variables, such as intentions and anxiety. As a result, two latent covariates were constructed: (1) self-related beliefs and (2) motivation. Items on these two latent covariates were scored on a four-point scale and were estimated using a partial credit IRT model (PCM; Masters, 1982).

\subsubsection{Student Motivation as a Latent Covariate}

PISA 2012 included scales measuring intrinsic and instrumental motivation, and short-term and long-term intentions to address the student motivation for mathematics (OECD, 2013). In this study, the eight-item intrinsic and instrumental motivation scale was used as an indicator of student motivation. The mathematics intentions measure was not included in the analyses since its scale did not combine meaningfully with the intrinsic and instrumental motivation scale. The coefficient alpha values for the four-item intrinsic motivation subscale and the four-item instrumental motivation subscale were both .90 . The coefficient alpha for the eight-item student motivation scale was .92. Principal axis factoring yielded two factors that correlated .65 . The two factors explained $62 \%$ and $15 \%$ of the variance, respectively. The factor loadings from an oblimin rotation with Kaiser normalization indicated that items on the instrumental motivation scale loaded on the first factor, and items on the intrinsic motivation scale loaded on the second factor. 


\subsubsection{Self-Related Beliefs as a Latent Covariate}

Self-efficacy and self-concept are commonly used measures of self-beliefs in academic motivation research (Pajares \& Schunk, 2001). Self-efficacy is described as a conviction or belief about one's ability to cope with certain tasks and self-concept is described as one's overall perception of his or her personal attributes evaluated by using continuous selfevaluation (OECD, 2013). A composite scale of self-beliefs was created by combining these two scales. The self-efficacy scale included eight items; the self-concept scale had five items. The coeffcieint alpha for the eight-item self-efficacy subscale was .86, and the coefficient alpha for the five-item self-concept subscale was .90. The coefficient alpha for the 13-item self-beliefs scale was .90. Principal axis factoring indicated two factors. The correlation between the two factors was .60. The factors explained $46 \%$ and $12 \%$ of the variance, respectively. Factor loadings from an oblimin rotation with Kaiser normalization indicated that the items of the selfefficacy scale loaded on the first factor, and items of the self-concept scale loaded on the second factor.

\subsubsection{Association between Covariates and Mathematics Achievement}

The association between the covariates and the mathematics achievement is shown in Table 1. The manifest variables of PISA 2012 (i.e., 31 manifest variables) together explained only $26 \%$ of the variability in the mathematics achievement scores. Self-beliefs, on the other hand, explained $29 \%$ of the variance in mathematics achievement by itself.

Table 1. Association between the Covariates and the Mathematics Achievement.

\begin{tabular}{lcccc}
\hline & $\begin{array}{c}\text { Mathematics } \\
\text { achievement }\end{array}$ & $\begin{array}{c}\text { Index for the number } \\
\text { of books at home }\end{array}$ & Motivation & Self-beliefs \\
\hline Mathematics achievement & 1.000 & $.359^{* *}$ & $.253^{* *}$ & $.535^{* *}$ \\
Index for the number of & & 1.000 & $.065^{* *}$ & $.158^{* *}$ \\
books at home & & & 1.000 & $.576^{* *}$ \\
Motivation & & & 1.000 \\
Self-beliefs & & & & \\
\hline
\end{tabular}

** Correlation is significant at the 0.01 level (2-tailed).

Two linear regression analyses were done to predict mathematics achievement score. In Study A (i.e., covariates that did not have a significant interaction), the index of the number of books and self-beliefs were used as predictors. In Study B, motivation and self-beliefs were used as predictors. The regression for Study A did not yield a significant interaction between number of books and self-beliefs $(\beta=-0.028, p=.639)$. The variables together explained $36 \%$ of the variance in mathematics achievement. In Study B, the covariates did have a significant interaction. The additive regression model, that is, the model with no interaction term for prediction of mathematics achievement using the self-belief and motivation scores, yielded a negative coefficient for motivation $(\beta=-0.138, p<.001)$ and a positive coefficient for selfbeliefs $(\beta=0.611, p<.001)$. Adding motivation to the model along with self-beliefs improved the relationship between self-beliefs and mathematics achievement but changed the sign of the coefficient for motivation indicating a suppression effect and, therefore, collinearity (Cohen, Cohen, West, \& Aiken, 2003) between the variables. In this instance, motivation acted like a suppressor variable, as it had a weak positive correlation with mathematics achievement ( $r$ $=.21$ ) but a relatively strong correlation with self-beliefs $(r=.57)$. In addition, as suggested by Cohen et al. (2003), this resulted as the correlation between mathematics achievement and motivation was less than the product of the correlation between mathematics achievement and 
self-beliefs, and the correlation between the motivation and the self-beliefs (e.g., . $21<.53 \times .57$ $=.30)$.

Sequential regression analysis (also known as residual regression analysis) was used to account for the shared variance between the variables in the context of collinearity in the data. In this analysis, motivation was determined to be the important variable. Thus, self-beliefs was regressed against motivation. Self-beliefs was replaced with the residuals from this regression since the residuals represent the independent contribution of self-beliefs after accounting for motivation (Graham, 2003). In study B, therefore, motivation, the residuals that represented self-beliefs and the interaction of these two were used to predict mathematics achievement.

The initial analyses for covariate selection analyses showed that the effect sizes for motivation $(\beta=0.197, p<.001)$ and number of books at home $(\beta=0.292, p<.001)$ were smaller than that for self-beliefs $(\beta=0.506, p<.001)$. The interaction between the index of the number of books at home and self-beliefs was not significant $(\beta=-0.028, p=.639)$. The interaction between motivation and self-beliefs was significant, although it had only a relatively small effect size ( $\beta$ $=0.062, p=.026)$ using Cohen's (1988) rules of thumb. Therefore, the anticipated effects of the motivation and number of books at home on latent class membership were also smaller relative to the self-beliefs. Small coefficients (e.g., approximately zero) from MRM-Cov were expected for the number of books at home and self-beliefs interaction given that it was insignificant.

\subsubsection{Estimation of the Model Parameters}

Estimation of the model parameters for each model was done using Markov chain Monte Carlo (MCMC) as implemented in the computer software OpenBUGS (Lunn, Spiegelhalter, Thomas, \& Best, 2009) (see Appendix A). The convergence of the model parameter estimates was assessed using three indices. Auto-correlations were examined as one indicator of MCMC convergence. In addition, the Monte Carlo error (MC error) for each posterior estimate was examined to determine if it was less than or equal to $5 \%$ of the standard deviation. Finally, the Heidelberger and Welch (1983) convergence diagnostic was used. Based on these indices, burnin was determined to be $10 \%$ of the total of 30,000 iterations for each model except for the model with the number of books at home and the model with number of books at home and beliefs as covariates. History plots suggested a burn-in period of 5,000 and 4,000 for these latter two models, respectively.

\subsubsection{Estimation of the Self-Beliefs and Motivation Scale Scores}

The PCM was used for estimating self-beliefs and motivation. MC errors, Heidelberger and Welch (1983) and Geweke (1992) convergence diagnostics were used to inform convergence. The burn-in for the PCM was 30,000 iterations with a total of 150,000 post-burn-in iterations for estimation of self-beliefs. As some parameters had high autocorrelations, the chain was thinned to every 10th iteration resulting in 12,000 post-burn-in iterations used to obtain the posterior estimates. For motivation, the burn-in was 45,000 of a total of 225,000 iterations. The chain was thinned to every 10 th iteration to reduce autocorrelations, resulting in 18,000 postburn-in iterations.

\section{RESULT / FINDINGS}

\subsection{MRM Analysis of the Mathematics Achievement Data}

Schwarz's (1978) Bayesian information criterion (BIC) and Akaike's (1974) information criterion (AIC) were used to inform determining the number of latent classes in the models. BIC and AIC both suggested three latent classes for all models in both Study A (see Appendix B) and in Study B (see Appendix C). 
To compare item parameter estimates between different models, mean and sigma equating (Marco, 1977) was used to transform the scale of models with a covariate to the scale of the model without a covariate. Additional transformation was not required for comparisons of the latent classes within the same model since the item parameters were mean centered within each class (e.g., Choi, 2014).

\subsection{Results from Study A--Non-Interacting Covariates}

In Study A (i.e., covariates that did not have a significant interaction), MRM model with the index of the number of books as a covariate (MRM-Cov-Books), MRM model with self-beliefs as a covariate (MRM-Cov-Self-Beliefs), and MRM models with the index of the number of books and self-beliefs as covariates with and without an interaction term (MRM-Cov-SelfBeliefs\&Books) were estimated.

Squared errors within each class were calculated for comparing item parameter estimates from the model with a covariate (MRM-Cov) to the model without a covariate (MRM). In this study, the MRM was the baseline model. Squared errors were compared by taking square of differences between item parameter estimates from MRM model and item parameter estimates from MRM-Cov models for each latent class.

A factorial ANOVA was done to compare the log-transformed squared errors for item parameter estimates between the MRM-Cov models. The equal variances assumption was met using Levene's test $(F(11,420)=0.827, p=.613)$. ANOVA results yielded a significant interaction between model type and latent class $(F(6,420)=5.924, p<.001)$, with a small to moderate size eta-squared value of .065 based on Cohen's (1988) rules of thumb.

Pairwise comparisons of log-transformed squared errors for item parameter estimates between MRM-Cov models using Tukey's HSD procedure did not yield differences in mean squared error (MSE) values between the models for Class 1. This indicated the item parameter estimates from MRM-Cov models were similar to each other for Class 1. For Class 2 and Class 3, MSE values from MRM-Cov-Books were similar to the MSE values from MRM model, and smaller than the MSE values from the remaining MRM-Cov models. The MSE values from these remaining MRM-Cov models, on the other hand, were not different than each other. Similarly, the item parameter estimates from MRM-Cov-Books model were different than the item parameter estimates from the remaining MRM-Cov models, and the item parameter estimates from these remaining MRM-Cov models were similar to each other. This pattern was more evident in Class 3 than in Class 2. For all classes, the additive model without interaction and the model with interaction resulted in similar item parameter estimates.

A factorial ANOVA was conducted on the posterior standard deviations of item parameter estimates from different models to investigate whether a particular pattern existed for standard errors of item parameter estimates. A Box-Cox transformation $(\lambda=-0.656)$ was applied to the standard deviations, as implemented in the R package MASS (Venables \& Ripley, 2002). Levene's test $(F(14,525)=0.996, p=.456)$ suggested equal variances. ANOVA results indicated a significant interaction between model type and latent class $(F(8,525)=7.695, p$ $\left.<.001, \eta^{2}=.050\right)$.

Pairwise comparisons of the posterior standard deviations of item parameter estimates were done using bootstrapping method with 10,000 samples because the cell means after Box-Cox transformation were not interpretable. For Class 1 and Class 2, the standard errors of item parameter estimates were similar from the different models. In Class 3, the standard errors of item parameter estimates were similar for the MRM and MRM-Cov-Books models. The standard errors of item parameter estimates were similar for the remaining models. The standard errors from the latter group of models were smaller than the standard errors from the former group of models. 
The mean ability for Class 2 was fixed to zero for model identification, for each model. Class means for the different models were similar for Class 1 . For Class 3, the class means from MRM and MRM-Cov-Books were similar to each other, and the class means from the rest of the models were similar to each other, although the differences in class means were trivial.

The mixing proportions did not exhibit a substantial covariate effect on the proportion of examinees in different classes for Class 1 as the mixing proportions from the different models were similar to each other. On the other hand, there was a clear pattern of effect for Class 2 and Class 3. This was similar to the effect observed for the mean ability estimates of Class 3 . Specifically, the proportions of students in each class were similar for MRM and MRM-CovBooks, and the proportions of students in each class were similar for the remaining three models. Incorporating self-beliefs in the MRM model as a covariate, or incorporating selfbeliefs and the index of the number of books at home together with or without interaction resulted in an approximately $12 \%$ decrease in Class 2 membership and a $12 \%$ increase in the Class 3 membership. However, this did not result in a considerable change in membership to Class 1.

Coefficients from different models indicated that covariates did provide information for describing the latent classes (see Table 2). That is, the positive coefficients from MRM-CovBooks showed that the students were more likely to belong to Class 1 and Class 3 as the number of books at home increased. The smaller coefficient for Class 1 indicated a smaller probability of being a member in this class as the number of books increase, compared to Class 3. Smaller coefficients also indicated that the number of books had a smaller effect size for predicting the class membership. The coefficients from the MRM-Cov-Self-Beliefs model also indicate that the students with higher self-related beliefs scores were less likely to belong to Class 1, and more likely to belong to Class 3 . The exponents of the coefficients provide a measure of effect size in terms of odds ratios to indicate the effect of covariates on the latent class membership. The effect size for belonging to Class $3(\exp (2.908))$ was higher relative to belonging to Class $1(\exp (-0.719))$.

Inclusion of number of books at home and self-beliefs as the covariates in MRM without an interaction term yielded negative coefficients for both covariates for Class 1 and positive coefficients for Class 3. In other words, controlling for the number of books at home, the students with higher self-beliefs were less likely to be member of the Class 1 and more likely to belong to Class 3. Similarly, controlling for self-beliefs, students possessing higher number of books at home were less likely to belong Class 1 and more likely to belong Class 3. For Class 1 , the coefficients for number of books at home and self-beliefs were similar to each other, which implies lacking of a differential covariate effect for this class. For Class 3, the effect size for self-beliefs controlling for number of books at home was $11.393(=\exp (2.958) / \exp (0.525))$ times the effect size for number of books at home controlling for self-beliefs. This exhibited a differential covariate effect for this class. Controlling for the effects of number of books did not cause a substantial change in coefficients of self-beliefs for both Class 1 and Class 3, compared to MRM-Cov-Self-Beliefs model. On the other hand, controlling for the self-beliefs caused a decrease in the coefficients of number of books at home for both Class 1 and Class3, compared to the MRM-Cov-Books model. This was consistent with results indicating smaller effect size for the number of books $(\beta=0.292)$ and a larger effect size for self-beliefs $(\beta=0.506)$ for predicting the mathematics achievement. Adding an interaction term to the MRM-Cov model with number of books at home and the self-beliefs did not result in a substantial change in the coefficients compared to the model without interaction. Further, the coefficient for the interaction term was approximately zero, consistent with the non-significant interaction term for predicting mathematics raw scores $(\beta=-0.028, p=.639)$. 
Table 2A. Coefficients from Different Models for Study A.

\begin{tabular}{ccccc}
\hline & \multicolumn{2}{c}{ MRM-Cov-Books } & \multicolumn{2}{c}{ MRM-Cov-Self-Beliefs } \\
\hline Class & Intercept & Books & Intercept & Beliefs \\
\hline 1 & -0.288 & 0.904 & -0.804 & -0.719 \\
2 & 0.000 & 0.000 & 0.000 & 0.000 \\
3 & -2.813 & 1.400 & -3.804 & 2.908 \\
\hline
\end{tabular}

Table 2B. Coefficients from Different Models for Study A.

\begin{tabular}{cccccccc}
\hline & \multicolumn{3}{c}{$\begin{array}{c}\text { MRM-Cov-Self-Beliefs\&Books } \\
\text { (No Interaction) }\end{array}$} & \multicolumn{3}{c}{$\begin{array}{c}\text { MRM-Cov-Self-Beliefs\&Books } \\
\text { (Interaction) }\end{array}$} \\
\hline Class & Intercept & Books & Beliefs & Intercept & Books & Beliefs & Books*Beliefs \\
\hline 1 & 0.720 & -0.793 & -0.742 & 0.679 & -0.791 & -0.674 & -0.041 \\
2 & 0.000 & 0.000 & 0.000 & 0.000 & 0.000 & 0.000 & 0.000 \\
3 & -5.252 & 0.525 & 2.958 & -5.574 & 0.631 & 3.231 & -0.094 \\
\hline
\end{tabular}

The MRM and MRM-Cov-Books had a high agreement of $93 \%$ for latent class assignment. The agreement between MRM and MRM-Cov-Self-Beliefs was $85 \%$, and the agreement between MRM and MRM-Cov-Self-Beliefs\&Books was $84 \%$ and $84 \%$ with and without interaction terms, respectively. The agreements in class membership for these pairs of models were similar to each other and smaller than the agreement between the classifications from the MRM and MRM-Cov-Books models. Overall, the results suggest that the covariates exhibited a considerable effect on class membership as the agreement between the MRM and MRM-Cov models changed considerably, depending on the covariate in the model.

\subsection{Results from Study B--Interacting Latent Covariates}

In Study B (i.e., covariates did have a significant interaction), MRM model with motivation as a covariate (MRM-Cov-Motivation), MRM model with self-beliefs as a covariate (MRM-CovSelf-Beliefs), and MRM models with motivation and self-beliefs as covariates with and without an interaction term (MRM-Cov-Self-Beliefs\&Motivation) were estimated.

Squared errors for item parameter estimates from the MRM model and the MRM-Cov models were calculated for each latent class as the square of the difference between item parameter estimates from the MRM model and from each of the MRM-Cov models. Factorial ANOVA analysis of natural log-transformed squared errors was conducted for comparing the item parameter estimates from MRM-Cov models. Homogeneity of variances assumption was met based on Levene's test $(F(11,420)=1.042, p=.408)$. Results indicated a non-significant interaction between type of model and latent class $(F(6,420)=0.810, p=.562)$. The main effects, however, were significant for both model type $\left(\mathrm{F}(3,420)=20.020, p<.001, \eta^{2}=.107\right)$ and latent class $\left(F(2,420)=38.270, p<.001, \eta^{2}=.136\right)$, albeit with only moderate effect sizes. Pairwise comparisons of MRM-Cov models using Tukey's HSD procedure did not yield significant differences in MSE values between the models for Class 1. For Class 2 and Class 3, MSE values between the MRM and the MRM-Cov-Motivation models were different than those between the MRM and the remaining MRM-Cov models. The MSE values from the remaining MRM-Cov models, however, were not different and were larger than those between the MRM and the MRM-Cov-Motivation model. 
In other words, the item parameter estimates from MRM-Cov models were similar to each other for Class 1. For Class 2 and Class 3, however, the item parameter estimates from MRM-CovMotivation were more similar to those from the MRM model than they were to the remaining MRM-Cov models. Similarly, the item parameter estimates from these three remaining MRMCov models were similar to each other. For all classes, the additive model (i.e., without interaction) and the model with interaction resulted in similar item parameter estimates.

An ANOVA analysis was conducted on the posterior standard deviations of item parameter estimates from different models. A Box-Cox transformation $(\lambda=-0.667)$ was applied to the standard deviations. Levene's test indicated that the equal variance assumption was met $(F(14$, $525)=0.943, p=.511)$. Results indicated a significant interaction between model type and latent class $\left(F(8,525)=6.224, p<.001, \eta^{2}=.041\right)$. Pairwise comparisons between posterior standard deviations of item parameter estimates were done using bootstrapping with 10,000 samples. For Class 1 and Class 2, the standard errors of item parameter estimates were similar from the different models. In Class 3, the standard errors of item parameter estimates were similar for MRM and MRM-Cov-Motivation models. Likewise, the standard errors of item parameter estimates were similar for the three remaining models. The standard errors from the latter group of models were smaller than the standard errors from the former group of models.

The mean ability for Class 2 was fixed to zero for model identification, for each model. The class means from the different models were similar for Class 1. For Class 3, class means for ability appeared to be more alike for the MRM and MRM-Cov-Motivation models compared to class means for rest of the models, although the differences in class means were negligible.

The mixing proportions suggest that the proportion of students were similar across the models for Class 1. For Class 2 and Class 3, the mixing proportions were similar for MRM and MRMCov-Motivation and for the three remaining models. All three of these remaining models assigned more than half of the students to the second class. The inclusion of motivation as a covariate in the model classified roughly $4 \%$ of the students from Class 1 and Class 2 into Class 3 compared to the MRM. Incorporating self-beliefs in the MRM shifted approximately $12 \%$ of the students from Class 2 to Class 3. Adding self-beliefs and motivation together to the MRM model with or without an interaction term shifted about $12 \%$ of the students from Class 2 to Class 3.

Coefficients from different models exhibited a covariate effect for helping to characterize the latent classes (see Table 3). The positive coefficients from the MRM-Cov-Motivation model indicated that students were more likely to belong to Class 3 as the motivation score increases. The smaller coefficient for Class 1 , on the other hand, indicated that the effect size for motivation was small. The coefficients in the MRM-Cov-Self-Beliefs model were negative for Class 1 and positive for Class 3 indicating examinees were less likely to belong to Class 1 and more likely to belong to Class 3 as their self-beliefs score increased. The model with both motivation and self-beliefs as covariates without an interaction term yielded a roughly zero coefficient for motivation and a negative coefficient for self-beliefs in Class 1 . In other words, controlling for the motivation, the students with higher self-beliefs were less likely to be members of Class 1 . On the other hand, controlling for self-beliefs, motivation did not show sufficient predictive power to estimate group membership for Class 1 . The positive coefficients for Class 3 indicated that the students were more likely to be a member of Class 3 as either motivation or self-beliefs increased after controlling for the other variable. This tendency for self-beliefs was $8.551(=\exp (3.284) / \exp (1.138))$ times the tendency for motivation in odds ratio.

The model with motivation, self-beliefs and their interaction yielded coefficients different than zero for the interaction in Class 1 and Class 3. This was expected since the previous regression analysis yielded a significant interaction term between motivation and self-beliefs for predicting 
mathematics raw scores $(\beta=0.062, p=.026)$. For this model, controlling for self- beliefs and taking the interaction term into account, motivation did not show sufficient predictive power to estimate group membership for Class 1 . Controlling for motivation and taking the interaction term into account, students with higher self-beliefs were less likely to be members of Class 1 . Controlling for self- beliefs and taking the interaction term into account for Class 3, students with higher motivation scores were more likely to be members of this class. Similarly, students were more likely to be members of Class 3 as the self-beliefs increased. Controlling for the effects of motivation in the models with or without interaction, the coefficients for self-beliefs changed compared to the MRM-Cov-Self-Beliefs model in both Classes 1 and 3. Controlling for self-beliefs, on the other hand, did not cause a substantial change in the coefficients of motivation for Class 1 in the models with and without interactions compared to the MRM-CovMotivation. Controlling for self-beliefs, the coefficient for motivation differed somewhat for Class 3 both for the models with and without interactions. This suggested that using more than one covariate in the model helped explain class membership by taking into account the effect of the other covariate.

Table 3A. Coefficients from Different Models for Study B.

\begin{tabular}{ccccc}
\hline & \multicolumn{2}{c}{ MRM-Cov-Motivation } & \multicolumn{2}{c}{ MRM-Cov-Self-Beliefs } \\
\hline Class & Intercept & Motivation & Intercept & Beliefs \\
\hline 1 & -1.446 & 0.084 & -0.804 & -0.719 \\
2 & 0.000 & 0.000 & 0.000 & 0.000 \\
3 & -1.707 & 0.892 & -3.804 & 2.908 \\
\hline
\end{tabular}

Table 3B. Coefficients from Different Models for Study B.

\begin{tabular}{|c|c|c|c|c|c|c|c|}
\hline \multirow[b]{2}{*}{ Class } & \multicolumn{3}{|c|}{$\begin{array}{l}\text { MRM-Cov-Self-Beliefs } \\
\text { (Residualized) \& Motivation } \\
\text { (No Interaction) }\end{array}$} & \multicolumn{4}{|c|}{$\begin{array}{l}\text { MRM-Cov-Self-Beliefs (Residualized) \&Motivation } \\
\text { (Interaction) }\end{array}$} \\
\hline & Intercept & Motivation & Beliefs & Intercept & Motivation & Beliefs & Motivation*Beliefs \\
\hline 1 & -1.642 & 0.049 & -1.304 & -1.357 & 0.052 & -1.306 & -0.879 \\
\hline 2 & 0.000 & 0.000 & 0.000 & 0.000 & 0.000 & 0.000 & 0.000 \\
\hline 3 & -1.795 & 1.138 & 3.284 & -1.826 & 1.140 & 3.277 & -1.305 \\
\hline
\end{tabular}

Agreement in class membership between the MRM and MRM-Cov-Motivation was as high as 94\%. The agreement between the MRM and MRM-Cov-Self-Beliefs was $85 \%$ and between the MRM and MRM-Cov-Self-Beliefs\&Motivation was $84 \%$ and $84 \%$ with and without interaction terms, respectively. The agreements in class membership for these pairs of models were similar to each other, and smaller than the agreement between the MRM and MRM-Cov-Books models. The patterns in the class membership agreement were similar to previous results. That is, the agreement between the MRM and MRM-Cov-Motivation models was greater than between the MRM and the remaining models. Incorporating self-beliefs and motivation in the MRM model together with or without interaction resulted in an approximately $13 \%$ decrease in Class 2 membership and a 13\% increase in the Class 3 membership. However, this did not result in a considerable change in membership to Class 1 . Results indicated a covariate effect on class membership causing students to shift between classes. This was likely because the agreement between the MRM and MRM-Cov models changed considerably depending on the covariate in the model. 


\section{DISCUSSION and CONCLUSION}

This study was designed to investigate effects of use of a covariate in a mixture Rasch model (MRM) on latent class membership. In most testing situations, manifest variables such as demographic information can be obtained easily through short surveys following the administration of the test. The association between the manifest variables and the latent class variable, however, is generally moderate (e.g., Cohen \& Bolt, 2005). Similarly, manifest variables in this study were found to account for a relatively small portion of the variance in mathematics achievement, even though they were significant predictors. Latent variables, on the other hand, explained a greater proportion of the variance showing the potential of the latent variables to be the better predictors of latent class membership compared to manifest variables, although some latent variables may be more useful than the others. Results of this study were consistent with previous research that latent covariates were more likely to have stronger associations with the dimension(s) along which the latent classes form. Contrary to the manifest variables, latent variables were also useful in this study for constructing meaningful composite scores based on previous research.

The results showed that the manifest and latent covariates did not have an impact on the number of underlying latent classes extracted, however, they helped explain the characteristics of the latent classes. The covariates changed the latent class membership proportions, however, they did not indicate a strong effect on class ability means. Latent covariates were more useful for explaining the characteristics of latent class membership compared to manifest covariates. Using more than one covariate did help explain the group membership after controlling for the other covariate. The effects of the covariates on latent class membership and on item parameters were class specific. Substantial effects of covariates on item parameters returned smaller standard errors for the item parameter estimates.

Results of this study suggested that incorporating more than one covariate in a mixture Rasch model should consider possible interactions between the covariates. Study A included covariates without a significant interaction, while Study B included covariates with significant interaction, although the interaction in Study B had a relatively small effect size. The models with interaction terms did not exhibit an effect on latent class membership proportions that was different from that for models without an interaction term. The significant interaction term in Study B, however, did show an effect on the coefficients for predicting and explaining latent class membership. It can be noted that the findings from this study are based on the two example studies that used empirical data and, hence, may not have direct applicability to other data or other sets of available manifested and latent covariates. More investigations, including simulation studies for which some parameters can be fully manipulated by researchers, are in need to check the generalizability of the findings.

\section{ORCID}

Tuğba Karadavut (iD https://orcid.org/0000-0002-8738-7177
Allan S. Cohen (id) https://orcid.org/0000-0002-8776-9378
Seock-Ho Kim (i) https://orcid.org/0000-0002-2353-7826

\section{REFERENCES}

Akaike, H. (1974). A new look at the statistical model identification. IEEE Transactions on Automatic Control, 19, 716-723.

Bilir, M. K. (2009). Mixture item response theory-MIMIC Model: Simultaneous estimation of differential item functioning for manifest groups and latent classes. Unpublished doctoral dissertation. Florida State University. 
Bolt, D. M., Cohen, A. S., \& Wollack, J. A. (2002). Item parameter estimation under conditions of test speededness: Application of a mixture Rasch model with ordinal constraints. Journal of Educational Measurement, 39, 331-348.

Bonnet, G. (2002). Reflections in a Critical Eye: On the pitfalls of international assessment. Assessment in Education: Principles, Policy \& Practice, 9, 387-399.

Cho, S. J., Cohen, A. S., \& Kim, S.-H. (2013). Markov chain Monte Carlo estimation of a mixture item response theory model. Journal of Statistical Computation and Simulation, 83, 278-306.

Choi, Y. J. (2014). Metric identification in mixture IRT models. Unpublished doctoral dissertation. University of Georgia.

Choi, Y. J., Alexeev, N., \& Cohen, A. S. (2015). Differential item functioning analysis using a mixture 3-parameter logistic model with a covariate on the TIMSS 2007 mathematics test. International Journal of Testing, 15, 239-253.

Cohen, A. S., \& Bolt, D. M. (2005). A mixture model analysis of differential item functioning. Journal of Educational Measurement, 42, 133-148.

Cohen, J. (1988). Statistical power analysis for the behavioral sciences (2nd ed.). Hills-dale, NJ: Erlbaum.

Cohen, J., Cohen, P., West, S. G., \& Aiken, L. S. (2003). Applied multiple regression/correlation analysis for the behavioral sciences (3rd ed.). New York, NY: Routledge.

Dai, Y. (2013). A mixture Rasch model with a covariate: A simulation study via Bayesian Markov chain Monte Carlo estimation. Applied Psychological Measurement, 37, 375396.

Geweke, J. (1992). Evaluating the accuracy of sampling-based approaches to calculating posterior moments. In J. M. Bernardo, J. O. Berger, A. P. Dawid, \& F. M. Smith (Eds.), Bayesian statistics 4 (pp.169-193). New York, NY: Oxford Press.

Graham, M. H. (2003). Confronting multicollinearity in ecological multiple regression. Ecology, 84, 2809-2815.

Heidelberger, P., \& Welch, P. D. (1983). Simulation run length control in the presence of an initial transient. Operations Research, 31, 1109-1144.

Lunn, D., Spiegelhalter, D., Thomas, A., \& Best, N. (2009). The BUGS project: Evolution, critique and future directions. Statistics in Medicine, 28, 3049-3082.

Marco, G. L. (1977). Item characteristic curve solutions to three intractable testing problems. Journal of Educational Measurement 14, 139-160.

Masters, G. N. (1982). A Rasch model for partial credit scoring. Psychometrika, 47, 149-174.

OECD. (2013), PISA 2012 assessment and analytical framework: Mathematics, reading, science, problem solving and financial literacy. Paris, France: OECD Publishing. Retrieved from http://dx.doi.org/10.1787/9789264190511-en

OECD. (2014). PISA 2012 technical report. Paris, France: OECD Publishing. Retrieved from www.oecd.org/pisa/pisaproducts/PISA-2012-technical-report-final.pdf

Pajares, F., \& Schunk, D. H. (2001). Self-beliefs and school success: Self-efficacy, selfconcept, and and school achievement. In R. Riding \& S. Rayner (Eds.), Perception (pp. 239-266). London, England: Ablex.

Rost, J. (1990). Rasch models in latent classes: An integration of two approaches to item analysis. Applied Psychological Measurement, 14, 271-282.

Schwarz, G. (1978). Estimating the dimension of a model. Annals of Statistics, 6, 461-464.

Smit, A., Kelderman, H., \& van der Flier, H. (1999). Collateral information and mixed Rasch models. Methods of Psychological Research Online, 4, 1-13.

Venables, W. N., \& Ripley, B. D. (2002). Modern applied statistics with S (4th ed.). New York, NY: Springer. 
Appendix A. OpenBUGS syntax for the MRM-Cov Models.

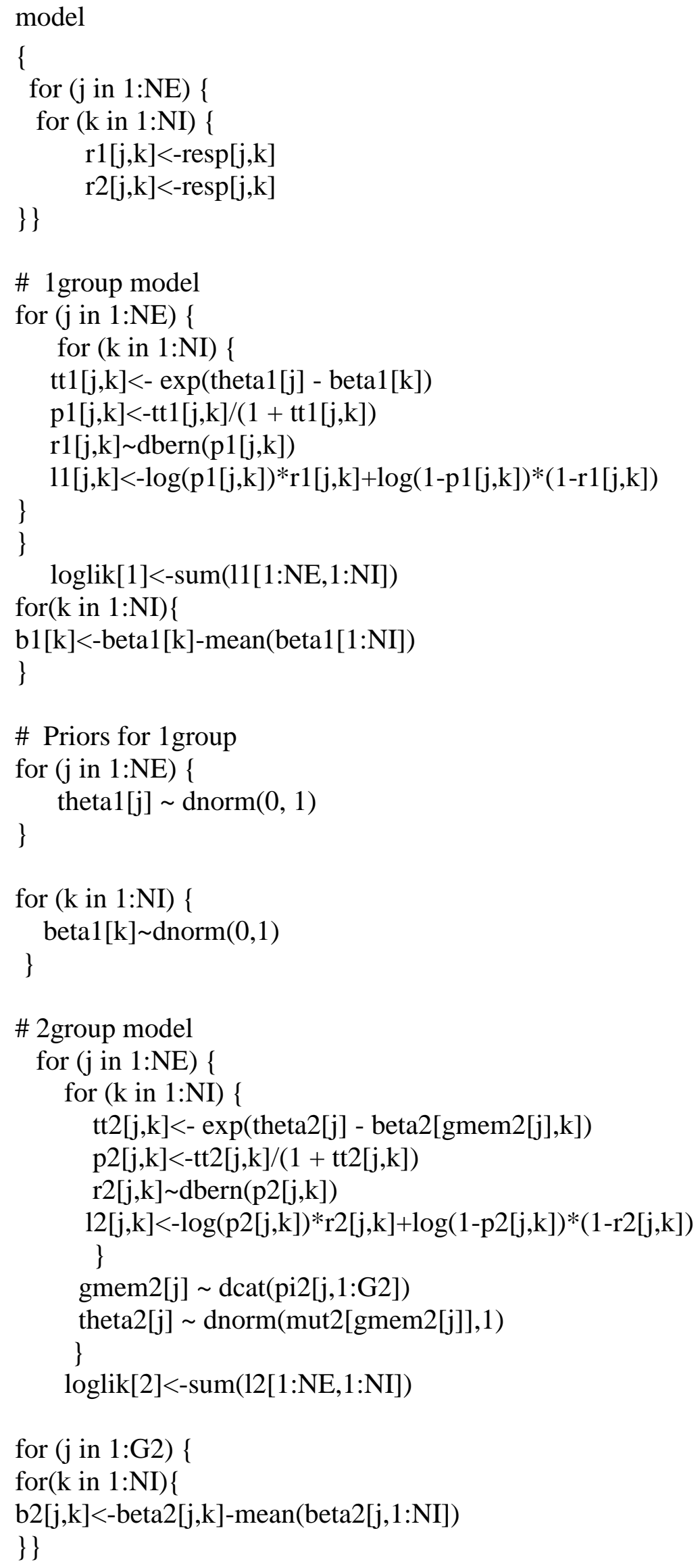




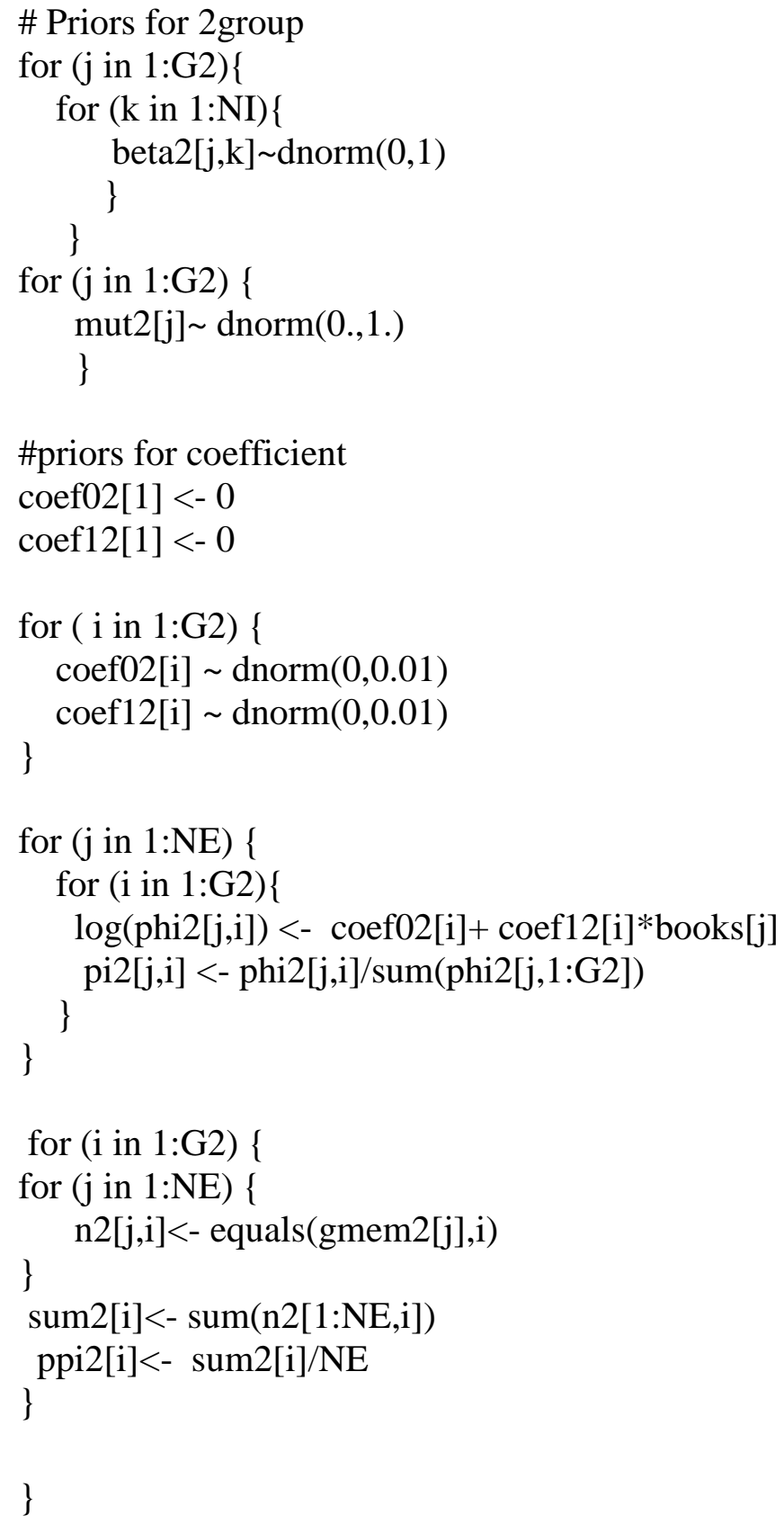


Appendix B1. Model Fit Indices for Study A.

\begin{tabular}{ccccccc}
\hline & \multicolumn{2}{c}{ MRM } & \multicolumn{2}{c}{ MRM-Cov-Books } & \multicolumn{2}{c}{ MRM-Cov-Self-Beliefs } \\
\hline $\begin{array}{c}\text { Number of } \\
\text { Classes }\end{array}$ & BIC & AIC & BIC & AIC & BIC & AIC \\
\hline 1 & 44510 & 44320 & 44510 & 44320 & 44510 & 44320 \\
2 & 43690 & 43300 & 43730 & 43340 & 43800 & 43410 \\
3 & $\mathbf{4 3 3 9 0}$ & $\mathbf{4 2 8 0 0}$ & $\mathbf{4 3 4 7 0}$ & $\mathbf{4 2 8 7 0}$ & $\mathbf{4 3 3 8 0}$ & $\mathbf{4 2 7 9 0}$ \\
4 & 43640 & 42850 & 43720 & 42930 & 43650 & 42860 \\
\hline
\end{tabular}

Note. AIC $=$ Akaike information criterion; $\mathrm{BIC}=$ Bayesian information criterion; the smallest information criterion index is in bold.

Appendix B2. Model Fit Indices for Study A.

\begin{tabular}{ccccc}
\hline & \multicolumn{2}{c}{$\begin{array}{c}\text { MRM-Cov-Self-Beliefs\&Books } \\
\text { (No Interaction) }\end{array}$} & $\begin{array}{c}\text { MRM-Cov-Self-Beliefs\&Books } \\
\text { (Interaction) }\end{array}$ \\
\hline $\begin{array}{c}\text { Number of } \\
\text { Classes }\end{array}$ & BIC & AIC & BIC & AIC \\
\hline 1 & 44510 & 44320 & 44510 & 44320 \\
2 & 43800 & 43410 & 43810 & 43410 \\
3 & $\mathbf{4 3 4 3 0}$ & $\mathbf{4 2 8 4 0}$ & $\mathbf{4 3 4 4 0}$ & $\mathbf{4 2 8 5 0}$ \\
4 & 43710 & 42920 & 44310 & 43520
\end{tabular}

Note. AIC $=$ Akaike information criterion; $\mathrm{BIC}=$ Bayesian information criterion; the smallest information criterion index is in bold.

Appendix C. Model Fit Indices for Study B.

\begin{tabular}{ccccccc}
\hline & MRM-Cov-Motivation & $\begin{array}{c}\text { MRM-Cov-Self- } \\
\text { Beliefs(Residualized)- } \\
\text { Motivation } \\
\text { (No Interaction) }\end{array}$ & \multicolumn{2}{c}{$\begin{array}{c}\text { MRM-Cov-Self- } \\
\text { Beliefs(Residualized)- } \\
\text { Motivation } \\
\text { (Interaction) }\end{array}$} \\
\hline $\begin{array}{c}\text { Number of } \\
\text { Classes }\end{array}$ & BIC & AIC & BIC & AIC & BIC & AIC \\
\hline 1 & 44510 & 44320 & 44510 & 44320 & 44510 & 44320 \\
2 & 43730 & 43340 & 43810 & 43420 & 43810 & 43420 \\
3 & $\mathbf{4 3 3 8 0}$ & $\mathbf{4 2 7 8 0}$ & $\mathbf{4 3 4 0 0}$ & $\mathbf{4 2 8 1 0}$ & $\mathbf{4 3 4 0 0}$ & $\mathbf{4 2 8 1 0}$ \\
4 & 43630 & 42840 & 43660 & 42870 & 43680 & 42890 \\
\hline
\end{tabular}

Note. AIC = Akaike information criterion; BIC = Bayesian information criterion; the smallest information criterion index is in bold. 